\title{
Automated HTS/HCS for antivirals using visual HIV full replication
} assays

Jean-Philippe Carralot ${ }^{1}$, Mi-Ri Kim², Philippe Kieffer-Kwon ${ }^{3}$, Sandra Corre ${ }^{3}$, Hwa Pyung Kim ${ }^{4}$, Junwon Kim5, Boris Lenseigne ${ }^{6}$, Changbok Lee ${ }^{2}$, Denis Fenistein ${ }^{6}$, Thierry Dorval ${ }^{6}$, Hyunrim $\mathrm{Oh}^{2}$, Sungjun Han ${ }^{4}$, Zaesung No ${ }^{5}$, Priscille Brodin ${ }^{1}$, Jonathan Cechetto ${ }^{2}$, Annette Boese ${ }^{3}$, Thierry Christophe ${ }^{2}$ and Peter Sommer*3

Address: ${ }^{1}$ Biology of Intracellular Pathogens Unit, Institut Pasteur Korea, Sampyeong-dong 696, Bundang-gu, Seongnam-si, Gyeonggi-do, Korea, ${ }^{2}$ Screening Technology \& Pharmacology Unit, Institut Pasteur Korea, Sampyeong-dong 696, Bundang-gu, Seongnam-si, Gyeonggi-do, Korea, ${ }^{3}$ Cell Biology of Retroviruses Unit, Institut Pasteur Korea, Sampyeong-dong 696, Bundang-gu, Seongnam-si, Gyeonggi-do, Korea, ${ }^{4}$ Drug Biology Unit, Institut Pasteur Korea, Sampyeong-dong 696, Bundang-gu, Seongnam-si, Gyeonggi-do, Korea, ${ }^{5}$ Medicinal Chemistry Unit, Institut Pasteur Korea, Sampyeong-dong 696, Bundang-gu, Seongnam-si, Gyeonggi-do, Korea and 'Image Mining Unit, Institut Pasteur Korea, Sampyeong-dong 696,

Bundang-gu, Seongnam-si, Gyeonggi-do, Korea

* Corresponding author

from Frontiers of Retrovirology: Complex retroviruses, retroelements and their hosts Montpellier, France. 21-23 September 2009

Published: 24 September 2009

Retrovirology 2009, 6(Suppl 2):P82 doi:10.1 I86/1742-4690-6-S2-P82

This abstract is available from: http://www.retrovirology.com/content/6/S2/P82

(c) 2009 Carralot et al; licensee BioMed Central Ltd.

There are currently 25 drugs belonging to 6 different inhibitor classes approved for the treatment of human immunodeficiency virus (HIV) infection. However, new anti-HIV agents and treatment strategies are still needed to confront the emergence of drug resistance and various adverse effects associated with long-term use of antiretroviral therapy and the inability to cure infected individuals. We developed visual, HIV full replication assays and implemented them in high-throughput compound $(\mathrm{n}=$ 200.000) and genome-wide siRNA screens, which allowed the identification of a few thousand novel small molecules with potent anti-retroviral activity and a few hundred host factors required for HIV infection, respectively. The identified compounds and host factors are opening unexplored avenues to novel antiviral drug and target discovery and validation, and should feed the drug development pipeline in the near future. 